\title{
Essai de dosage qualitatif et quantitatif de quelques pesticides organochlorés dans les produits laitiers par l'utilisation d'une précolonne en chromatographie gaz-liquide
}

\author{
par \\ M. DEMAIMAY, G. LAVOUE* et M. FEUILLAT \\ avec la collaboration technique de J.P. PIERROT \\ Laboratoire de Chimie et de Technologie agricole \\ Ecole Nationale d'Ingénieurs des Travaux Agricoles de Quetigny (21)
}

\section{Introduction}

La recherche et le dosage des pesticides dans les produits laitiers français ont fait l'objet ces dernières années de plusieurs communications et les auteurs n'ont pas manqué d'attirer l'attention des producteurs et des industriels sur l'importance du problème [1-2-3].

Les Etats-Unis se sont déjà dotés d'une réglementation officielle fixant une teneur limite en résidus pour les produits qu'ils importent. Il est probable que dans un avenir proche d'autres pays adopteront une conduite semblable et la législation française devra ellemême être précisée dans ce domaine.

$\mathrm{Si}$ à long terme le but à atteindre est d'éliminer les principales causes de contamination, il se pose dans l'immédiat le problème de pouvoir contrôler par l'analyse le plus grand nombre de produits possibles.

Deux méthodes sont actuellement pratiquées en France : la méthode officielle américaine (F.D.A.) [4] et la méthode de Goursaud [5]. Toutes deux relèvent de la chromatographie en phase gazeuse qui est une technique particulièrement bien adaptée aux microdosages.

La méthode que nous décrivons ci-dessous est une variante qui vise à faciliter la fréquence des contrôles industriels par une analyse plus simple, plus rapide et d'un coût moins élevé.

* Département chromatographie, Packard-Becker, Packard instrument (France). 


\section{I. - Principe de la méthode}

Les pesticides organochlorés étant des composés stables liposolubles, il nous est apparu intéressant de rechercher une méthode de dosage par injection directe en chromatographie en phase gazeuse de l'extrait lipidique total des produits laitiers, sans faire appel à la " délipidation partielle " par chromatographie sur Florisil. En effet, outre les manipulations supplémentaires que cette opération implique et le prix de revient élevé du produit, on peut toujours soupçonner un certain pourcentage de perte en pesticides.

L'extrait lipidique total renfermant les pesticides est donc injecté directement en phase gazeuse sur une colonne chromatographique qui comprend deux parties :

- La première appelée précolonne est garnie de Réoplex 400 qui fixe la matière grasse d'une façon spécifique.

- La seconde renferme une phase classique type $\mathrm{QF}_{1} 5$ p. 100 sur laquelle les pesticides sont analysés.

\section{II. - Matériel et réactifs}

\section{A. - Chromatographe en phase gazeuse}

- Le chromatographe utilisé est un appareil Packard, modèle 7409 équipé en outre de deux détecteurs à capture d'électrons, modèle utilisant des sources ionisantes au Nickel 63, d'une activitité de 15 millicuries.

- L'alimentation de ces détecteurs est à courant continu, la tension appliquée aux détecteurs est de 5 volts.

- L'injection des échantillons se fait directement en tête de colonne à l'aide d'une seringue Hamilton de $10 \mu \mathrm{l}$.

- Les colonnes spiralées, en verre pyrex, ont 1,80 m de long et $3 \mathrm{~mm}$ de diamètre interne.

- Débit du gaz vecteur (Argon - Méthane - 90/10, v/v) $40 \mathrm{ml} / \mathrm{mn}$.

- Elles sont remplies :

a) en tête de colonne : sur une longueur de $10 \mathrm{~cm}$ par du chromosol W traité imprégné de Réoplex 400 traité, produit par Packard Instrument (PB LG 71),

b) pour le reste de la colonne par du gaz chrom Q 100 - 120 mesh, imprégné à 5 p. 100 par du $\mathrm{QF}_{1}$, ou à 10 p. 100 par du DC 200.

N.B. - Précolonne et colonne sont séparées par un tampon de laine de verre. Ce dispositif permet de remplacer aisément le contenu de la précolonne lorsque celui-ci est usagé. Notons à cet égard 
qu'après une trentaine d'injections la précolonne utilisée est encore valable.

- La température de l'injecteur est de $230^{\circ} \mathrm{C}$ et celle du détecteur de $220^{\circ} \mathrm{C}$.

- La température de la colonne est de $210^{\circ} \mathrm{C}$ en analyse isothermique et peut varier de 190 à $230^{\circ} \mathrm{C}$ en analyse à température programmée à raison de $2^{\circ} \mathrm{C} / \mathrm{mn}$ lorsqu'on veut accélérer le temps d'élution du D.D.T. sur DC 200.

Remarque : Des essais menés jusqu'alors sur un détecteur au tritium ont donné des résultats beaucoup moins satisfaisants.

\section{B. - RÉACTIFS ET STANDARDS}

- Solvants purs produits par Merck.

- Pesticides étalons purs produits par Supelco.

\section{III. - Mode opératoire}

\section{A. - Contrôle DE LA VERRERIE ET DES SOlVANTS}

1) Verrerie : La verrerie utilisée au cours de l'extraction et des transferts de l'extrait est lavée à l'aide d'eau distillée additionnée de détergent Teepol, rincée à l'eau distillée et séchée à l'étuve.

Un échantillon de cette verrerie est prélevé sur chaque lot lavé, rincé à l'hexane distillé, ce solvant est concentré à $30^{\circ} \mathrm{C}$ sous vide et une partie aliquote injectée dans l'appareil. Nous avons pu ainsi vérifier que la méthode de lavage donnait un blanc ne recélant aucune trace de pesticides chlorés ou autres produits ayant le même temps de rétention que les pesticides que nous recherchons dans les produits laitiers.

2) Solvants : Tous les solvants ont été distillés. Les têtes et les queues de distillation ont été éliminées et la pureté de chacun des produits vérifiée par une analyse en chromatographie gazeuse.

B. - EXTRACTION DES RÉSIDUS DE PESTICIDES

1) Préparation de la matière grasse. Nous nous sommes appuyés sur la méthode d'analyse officielle américaine mise au point aux laboratoires de la Food and Drug Administration (F.D.A.).

\section{Extraction dans le fromage}

- Peser le fromage afin d'avoir dans l'échantillon environ $3 \mathrm{~g}$ de matières grasses après extraction des lipides.

- Mixer le mélange constitué par :

- le fromage,

- 2 g d'oxalate de sodium (contre les émulsions), 
- 2 fois le poids de fromage en sulfate de sodium anhydre,

- les solvants : acétone, éther de pétrole, éther éthylique dans les proportions $80-60-60 \mathrm{ml}$.

- Filtrer sur entonnoir, récupérer le filtrat.

- Laisser décanter le filtrat en présence de $300 \mathrm{ml}$ d'eau salée à 20 p. 1000 .

- Eliminer la phase aqueuse et faire passer la phase éthérée sur une colonne $(15 \times 1,5 \mathrm{~cm})$ de sulfate de sodium anhydre, afin de dessécher la matière grasse.

- Evaporer les solvants sous vide à $25-30^{\circ} \mathrm{C}$.

- Terminer l'évaporation des solvants en plaçant le ballon dans un bain marie à $50^{\circ} \mathrm{C}$.

Opérer sous hotte et introduire dans ce ballon un mince tube de verre ( $2 \mathrm{~mm}$ de diamètre) qui permet l'arrivée d'un courant d'azote favorisant l'évaporation des traces de solvants.

Extraction dans le lait

- Centrifuger $150 \mathrm{ml}$ de lait à 7000 tours pendant $15 \mathrm{mn}$.

- Reprendre le surnageant avec le mélange : acétone, éther de pétrole, éther éthylique ( $80-60$ - 60) y ajouter quelques gouttes d'oxalate de sodium. Laisser décanter ce mélange après y avoir ajouté $300 \mathrm{ml}$ d'eau salée à 20 p. 1000 .

- La suite des opérations est analogue à celle appliquée pour l'extraction des matières grasses dans le fromage.

2) La méthode Goursaud et al. a été utilisée comme méthode de référence.

\section{C. - Analyse chromatographioue}

1) Analyses d'échantillons de pesticides purs

a) Etablissement des courbes d'étalonnage pour les pesticides recherchés

Nature des pesticides : H.C.B., Dieldrine, H.C.H. alpha, Lindane, Heptachlore époxyde, Heptachlore, Aldrine.

A partir de $100 \mathrm{ml}$ d'une solution mère contenant $0,1 \mathrm{mg} / \mathrm{ml} \mathrm{du}$ pesticide considéré, plusieurs dilutions successives ont été effectuées afin d'obtenir des solutions étalons de concentration variant entre 0,05 et 0,50 p.p.m.

Cette gamme de sensibilité a été choisie, compte tenu des teneurs en pesticides détectables dans les conditions d'une analyse chromatographique de l'extrait lipidique total avec utilisation d'une précolonne et sans qu'il soit nécessaire de changer d'atténuation pour l'enregistrement des points limites 0,05 et 0,50 p.p.m. 
Après injection des différents standards sur l'ensemble précolonne et colonne, l'intégration de la surface des pics obtenu permet de tracer la courbe : surface du pic en fonction de la concentration.

L'ensemble des courbes est porté sur la figure 1.

Dans le domaine de sensibilité testé, une linéarité très satisfaisante a été obtenue avec tous les pesticides étudiés.

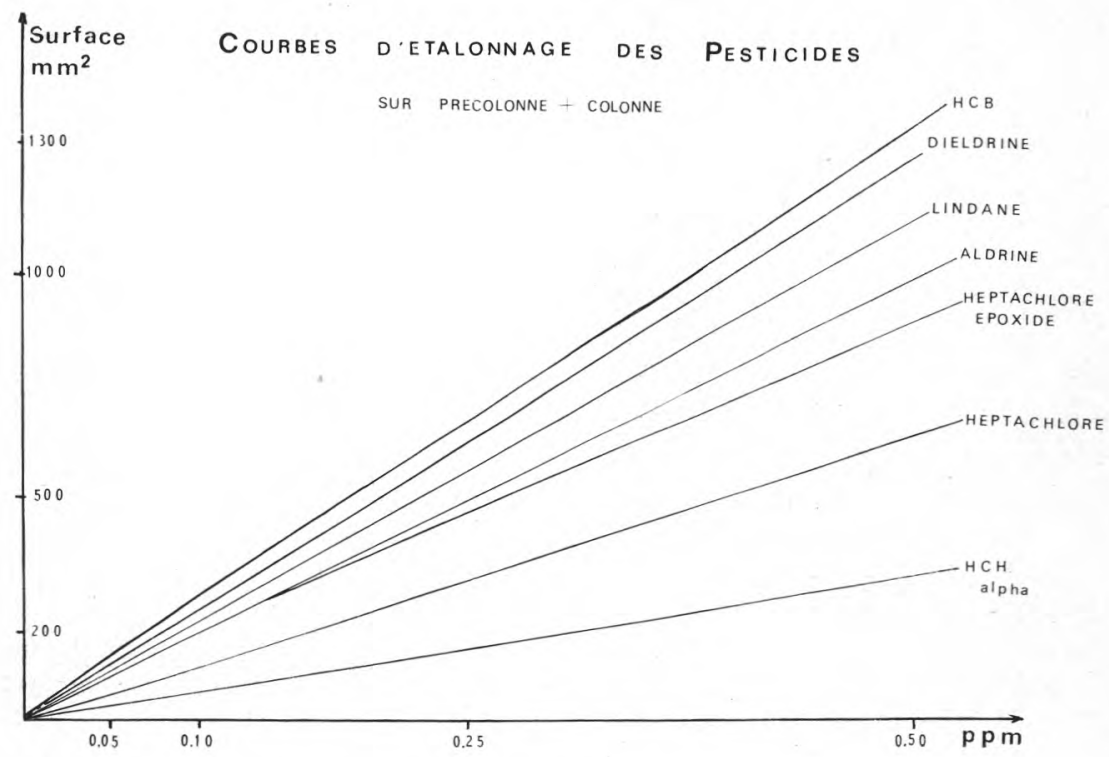

fig 1

TABLEAU 1

\begin{tabular}{l|c}
\hline \multicolumn{1}{c|}{ Pesticides } & $\begin{array}{c}\text { Distance de rétention relative à l'Aldrine } \\
\text { sur précolonne + colonne } \mathrm{QF}_{1} 5 \mathrm{p} .100\end{array}$ \\
\cline { 2 - 2 } H.C.B. & 0,46 \\
H.C.H. & 0,63 \\
Lindane & 0,83 \\
Heptachlore & 0,83 \\
Aldrine & 1 \\
Heptachlore époxide & 1,73 \\
Dieldrine & 2,7 \\
\hline
\end{tabular}


Notons que pour des concentrations en pesticides supérieures à 0,50 p.p.m., il est nécessaire d'effectuer une dilution afin de se situer dans le domaine des courbes établies.

b) Coefficient de rétention relatif : L'Aldrine est pris comme élément de référence et un coefficient de rétention relatif est calculé pour chaque pesticide.

Le tableau 1 donne les résultats des analyses faites sur précolonne et colonne $\mathrm{QF}_{1} 5$ p. 100.

c) Contrôle quantitatif : Un contrôle chromatographique nous a permis de vérifier qu'aucune perte apparente de pesticides ne semble avoir lieu lors du passage des différents produits sur la précolonne.

\section{2) Analyse d'extraits lipidiques de produits laitiers}

a) Choix des colonnes : Lorsqu'on veut rechercher ou doser séparément Heptachlore et Lindane, on utilise la colonne dont le support est imprégné de D.C. 200 à 10 p. 100, plutôt que le support imprégné de $\mathrm{QF}_{1}$ qui ne permet pas de séparer ces deux composés chlorés. La précolonne reste la même dans les deux cas.

b) Méthode d'injection : Les lipides issus des produits laitiers sont fluides à la température ambiante. Toutefois, afin d'éliminer les volumes morts de la seringue et d'injecter quantitativement tout le volume prélevé dans l'extrait total, on commence par prendre $2 \mu l$ d'Hexane distillé ; afin d'éviter que cet Hexane n'entre en contact direct avec l'échantillon au moment du prélèvement, on aspire ensuite $0,2 \mu \mathrm{l}$ d'air, puis les $5 \mu \mathrm{l}$ de l'échantillon avant d'injecter. Au moment de l'injection, l'Hexane chasse l'échantillon de l'aiguille et sa vaporisation rince parfaitement l'aiguille des traces de lipides absorbés sur sa paroi intérieure. Nous avons pu vérifier ainsi que la reproductibilité des injections est excellente et l'écart maximum observé d'une analyse à une autre inférieur à 3 p. 100 pour un pesticide donné.

c) Dans le cas de lipides concrets à la température ambiante, on peut envisager de mettre en solution ces lipides concrets (type suif de bœuf) dans l'Hexane et d'ajouter un standard interne approprié pour effectuer les dosages. Il suffit alors d'étalonner la réponse du standard interne par rapport à celle donnée par les autres pesticides.

\section{D. - RÉSultats}

\section{1) Rôle et importance de la précolonne pour la délipidation}

Dans le cas présent, nous avons cherché à supprimer la phase de délipidation par chromatographie sur Florisil d'un extrait de lipides totaux de produits laitiers contenant en outre les pesticides. 
PESTICIDES DANS UN ECHANTILLON DE FROMAGE

INJECTION DE MATIERES GRASS'S

QF-1 5\% SANS PRECOLONNE

ISOTHERMF $210^{\circ} \mathrm{C}$

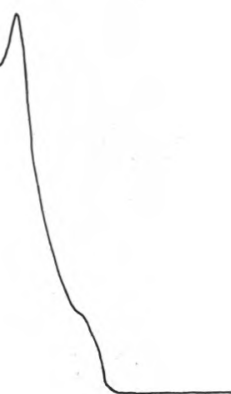

fig. 2

Il importe donc que la précolonne soit capable de bloquer spécifiquement l'élution des lipides injectés en phase gazeuse (esters de cholestérol, triglycérides, diglycérides, acides gras libres, monoglycérides et phospholipides) et de laisser passer qualitativement et quantitativement les pesticides recherchés par la capture d'électron. 


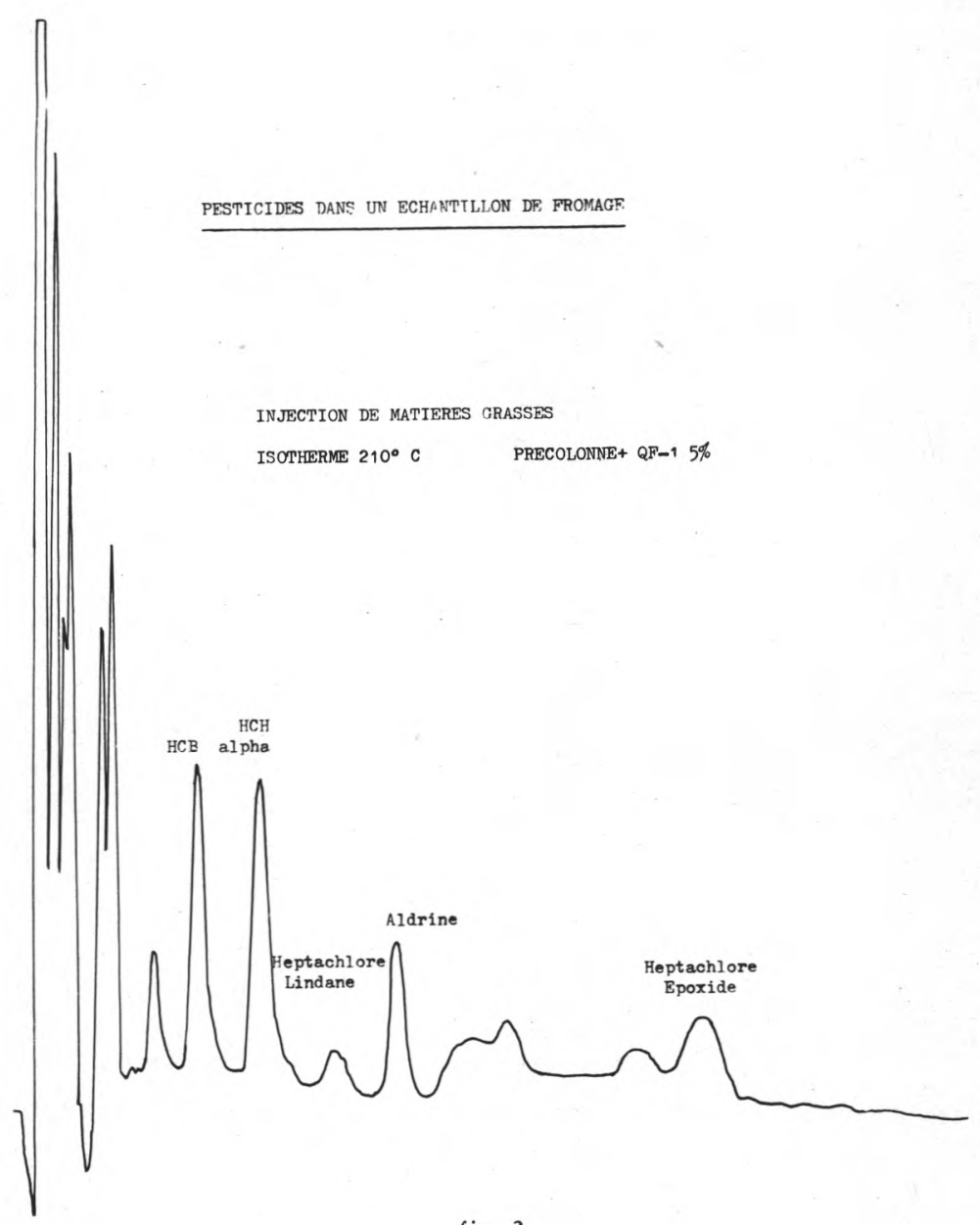

fig. 3

La figure 2 montre par l'importance du front d'injection qu'une colonne classique pour pesticides est incapable de jouer ce rôle.

Par contre, la figure 3 obtenue à partir de la même colonne munie de la précolonne montre à l'évidence le rôle joué par cette dernière. 
MÉMOIRES ORIGINAUX

51

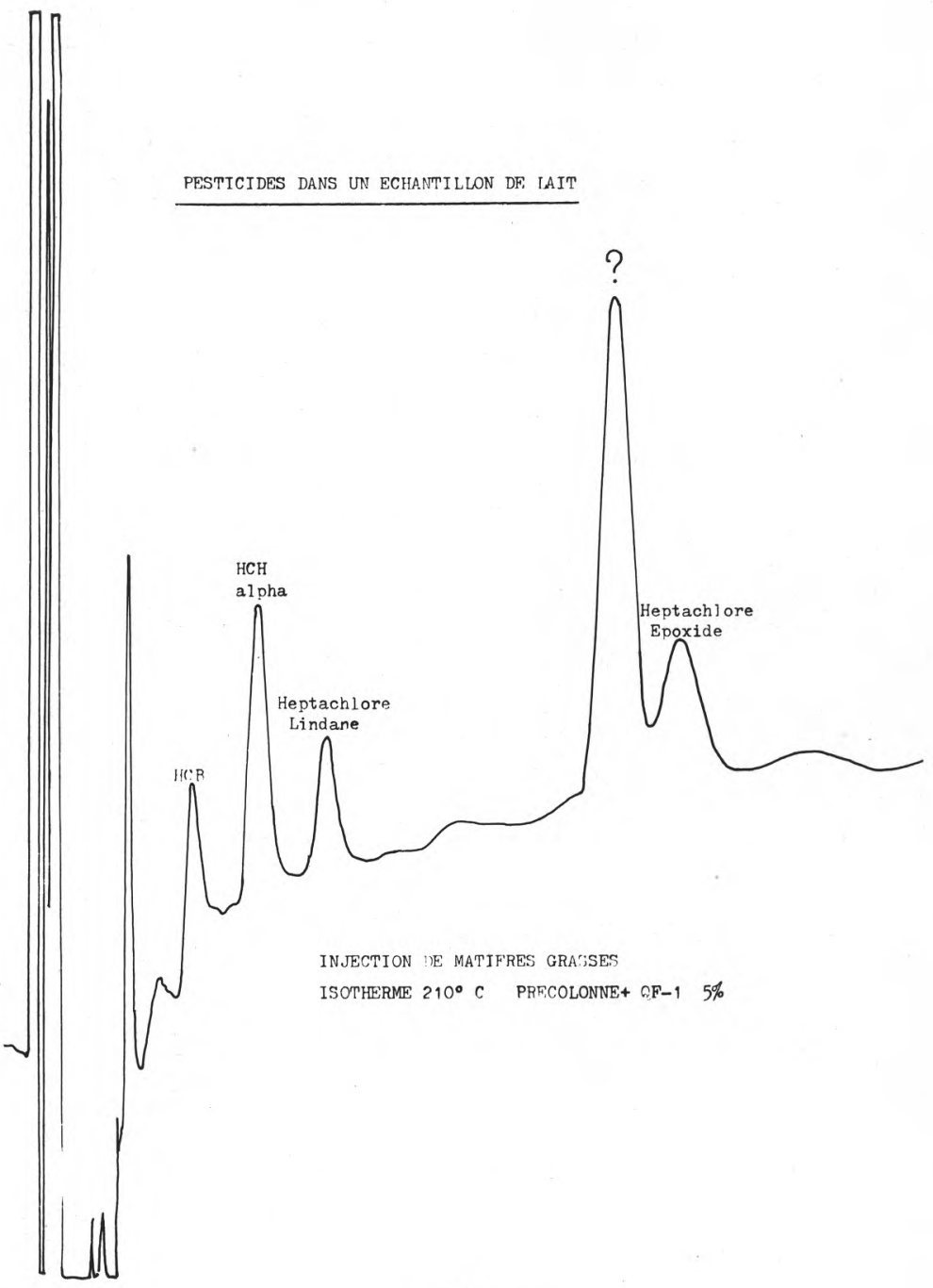

fig. 4

Un exemple d'analyse sur l'extrait lipidique total dans un échantillon de lait est donné par la figure 4.

2) Stabilité des échantillons

Nous avons pu observer en outre que le mélange des pesticides obtenus par la méthode Goursaud évoluait quantitativement dans le 


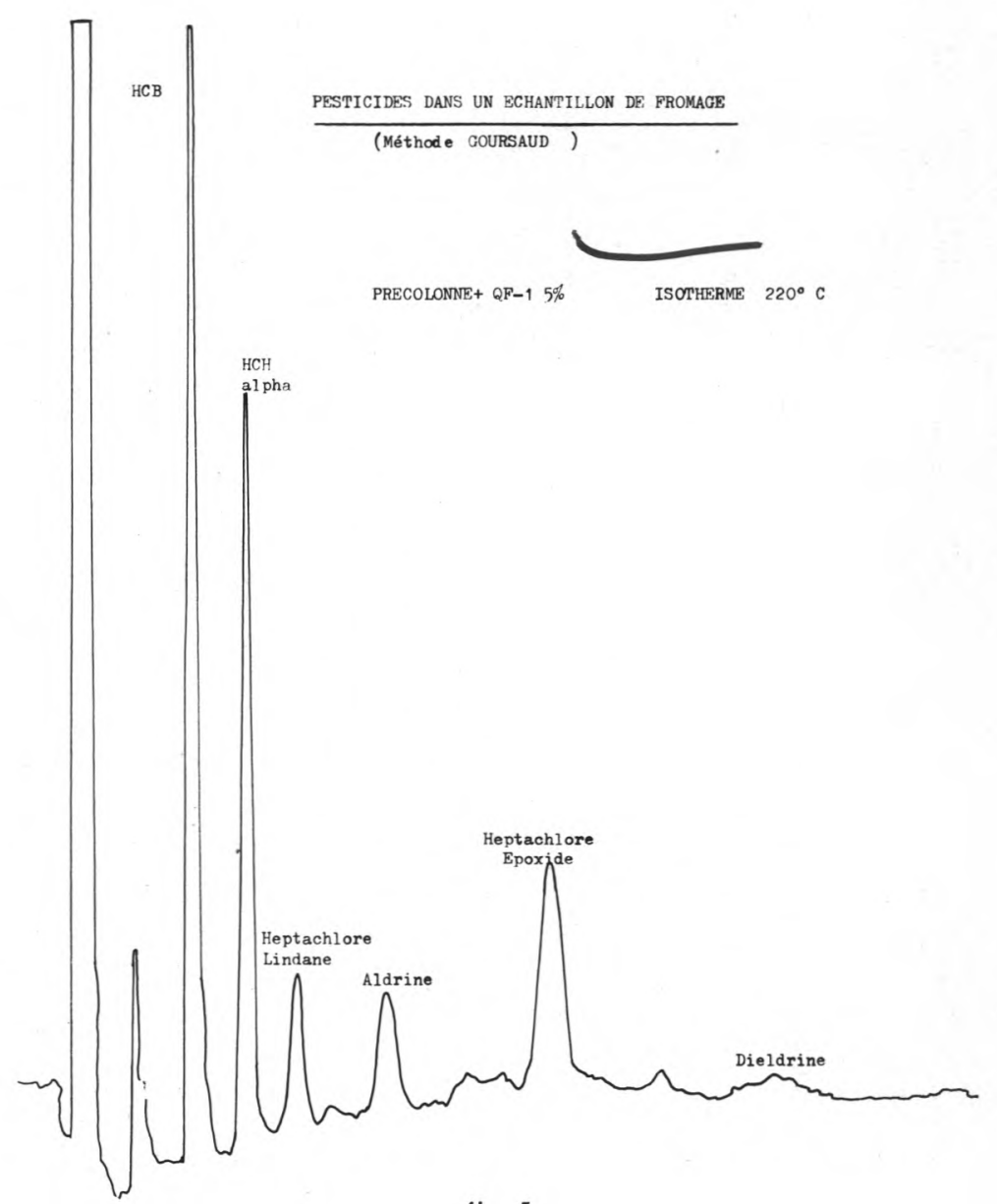

fig. 5

temps, si bien qu'après $8 \mathrm{j}$ environ à la température ambiante et à la lumière, un échantillon évolue de telle sorte qu'une nouvelle analyse donne des résultats très différents de ceux obtenus sur l'extrait frais. Par contre, stocké dans les mêmes conditions, l'extrait total (lipides et pesticides) que nous injectons sur la précolonne donne des résultats parfaitement reproductibles à $15 \mathrm{j}$ d'intervalle. 
Il semble donc que les lipides jouent un rôle de protection vis-à-vis des pesticides qu'ils contiennent.

\section{3) Etude comparative avec la méthode de référence}

Toutefois, nous avons observé parfois des différences quantitatives (fig. 3 et 5) sans que nous puissions mettre en cause plus particulièrement l'une ou l'autre méthode.

\section{$R$ és u m é}

Nous avons pu montrer que l'utilisation en série d'une précolonne et d'une colonne analytique en phase gazeuse permet d'envisager la recherche des pesticides chlorés par injection directe de l'extrait lipidique total. Un étalonnage satisfaisant a été effectué sur cet ensemble chromatographique qui permet d'analyser qualitativement et quantitativement les pesticides.

En outre, il apparaît que la stabilité des pesticides contenus dans l'extrait lipidique total est nettement supérieure à celle observée sur les pesticides extraits par la méthode Goursaud.

Enfin, il est évident que l'élimination du Florisil dans le traitement de l'échantillon simplifie le dosage et abaisse notablement son prix de revient.

L'analyse des extraits lipidiques de produits laitiers ne permet pas encore de tirer des conclusions définitives sur la reproductibilité de la méthode étant donné le petit nombre d'essais.

Une méthode comparative systématique est en cours actuellement dans une industrie laitière, afin de contrôler statistiquement la validité de la méthode au niveau du contrôle industriel.

\section{S u m m a ry}

We have be able to show that by using a precolumn connected to an analytical column in gas chromatography we can detect chlorinated pesticides by direct injection of total lipid extract. A satisfactory grading has been performed on this chromatographic set-up, which has enabled us to analyse pesticides both qualitatively and quantitatively.

It has been observed moreover that the stability of pesticides in the total lipid extract is more durable than in pesticides extracted by Goursaud process.

Of course, by treating the sample without using Florisil, dosing is made easier and cheaper. 
Owing to the small number of experiments carried out to date, analysing lipide extracts from dairy products does not yet allow us to draw final conclusions about reproductibility of the process.

A systematic comparative procedure is being carried out in a dairy plant in order to check statistically the validity of the process applied to control in industry.

\section{Bibliographie}

[1] Mestres (R.), Barthes (F.) et Mile Priu (M.) (Faculté de Pharmacie), Portal (F.) (Laboratoire de la Répression des Fraudes). - Recherche et dosage de l'Aldrine, de la Dieldrine et du D.D.T. dans le lait et le beurre. Société de Pharmacie de Monpellier, séance du 30-6-1966.

[2] Surveyor. - Les résidus de pesticides dans les laits et les produits laitiers. Revue Laitière Française, vol. 283, 1971.

[3] Casalis (J.), Luquet (F. M.) et Goursaud (J.). - Les résidus des pesticides dans les produits laitiers. Laboratoire de la chaire d'Industrie laitière de l'E.N.S.I.A.A. Communication faite aux Journées du Contrôle et de l'Analyse dans les Industries Alimentaires, Nantes le 16-17-18 mars 1971.

[4] Méthode d'analyse officielle Américaine (F.D.A.). Pesticides Analatycal Manuel, vol. I et II. U.S. Department of Health education and welfare. Food and drug administration.

[5] Goursaud (J.), Luquet (F. M.) et Casalis (J.). - Méthode rapide de contrôle de la teneur en résidus de quelques pesticides organochlorés dans les produits laitiers. Le Lait, novembre-décembre 1968, $\mathrm{n}^{\circ} 479-480$.

Reçu pour publication en juillet 1971. 